\title{
Advanced Digital 3D Technology in the Combined Surgery-First Orthognathic and Clear Aligner Orthodontic Therapy for Dentofacial Deformity Treatment
}

\author{
Minh Truong Nguyen ${ }^{1,2}$, Tien Thuy $\mathrm{Vu}^{3,4}$, and Quang Ngoc Nguyen ${ }^{5, *(D)}$ \\ 1 Department of Odonto-Stomatology, Hanoi Medical University Hospital, Hanoi Medical University, \\ Hanoi 100000, Vietnam; nguyentruongminh@hmu.edu.vn \\ 2 Department of Oral and Maxillofacial Surgery, School of Dentistry, Hanoi Medical University, \\ Hanoi 100000, Vietnam \\ 3 Dental Biomaterials Science Program, Graduate School, Chulalongkorn University, Bangkok 10330, Thailand \\ 4 Department of Endodontics, Hanoi National Hospital of Odonto-Stomatology, Hanoi 100000, Vietnam \\ 5 Department of Computer Science and Communications Engineering, Faculty of Science and Engineering, \\ Waseda University, Tokyo 169-0051, Japan \\ * Correspondence: Vu.Th@student.chula.ac.th (T.T.V.); quang.nguyen@fuji.waseda.jp (Q.N.N.)
}

\section{check for} updates

Citation: Nguyen, M.T.; Vu, T.T.; Nguyen, Q.N. Advanced Digital 3D Technology in the Combined Surgery-First Orthognathic and Clear Aligner Orthodontic Therapy for Dentofacial Deformity Treatment. Processes 2021, 9, 1609. https:// doi.org/10.3390/pr9091609

Academic Editors: Leszek

Adam Dobrzański and

Joanna Dobrzańska

Received: 15 July 2021

Accepted: 3 September 2021

Published: 8 September 2021

Publisher's Note: MDPI stays neutral with regard to jurisdictional claims in published maps and institutional affiliations.

Copyright: (c) 2021 by the authors. Licensee MDPI, Basel, Switzerland. This article is an open access article distributed under the terms and conditions of the Creative Commons Attribution (CC BY) license (https:// creativecommons.org/licenses/by/ $4.0 /)$.

\begin{abstract}
Orthognathic surgery and orthodontic treatment are required for patients with dentofacial deformities to obtain an ideal facial esthetic with good functioning. Recently, characterized by the surgery-first approach, an integrated orthodontic-surgical treatment has been introduced as an emerging solution to dentofacial deformity treatment. The surgery-first approach is regarded to have less treatment time and quicker enhancement of a facial profile than the conventional orthodonticsurgical treatment. Moreover, the recent advances in computing and imaging have allowed the adoption of 3-dimensional (3D) virtual planning protocols in orthognathic surgery as well as digital orthodontic treatment, which enables a paradigm shift when realizing virtual planning properly. These techniques then allow the surgeon and orthodontist to collaborate, plan, and simulate the dentofacial deformity treatment before performing the whole procedure. Along this line, in this research article, we present an integrated treatment method for the realization of an effective deformity treatment. Specifically, we implemented the integrated 3D technique by combining it with the surgery-first orthognathic approach (SFOA) as a novel treatment method for the patients. The outcomes from the combined treatments of the patients with dentofacial deformity, in practice, have demonstrated that our proposed 3D technique in orthognathics and orthodontics using clear aligner therapy (e.g., Invisalign) can enhance the satisfactory level of the patient since the start of treatment then improve their quality of life. As a result, the combined techniques realize the novel integrated treatment method using 3D technology with the use of 3D imaging and modeling as a promising development trend of dentistry, which fits into the context of Dentistry 4.0 as a key enabler to the concept of sustainable dentistry development.
\end{abstract}

Keywords: surgery-first orthognathic approach (SFOA); digital orthodontic; dentofacial deformity; 3D deformity treatment; clear aligner

\section{Introduction}

For patients with severe skeletal jaw discrepancies, the combination of orthognathic surgery (OGS) with orthodontics is the treatment of choice that can both harmonize facial esthetics and restore functional occlusion [1]. Unfortunately, conventional presurgical orthodontics involves a lengthy decompensation period that worsens the facial appearance of the patients and exacerbates the malocclusion [2]. Thanks to the immediate enhancement of the facial profile and the faster treatment time compared to the conventional approach, the recent surgery first (SF) approach is gaining popularity in orthognathic surgery [3]. Unlike the traditional approach, as its name suggests, no pre-surgical orthodontics is required and 
the surgical procedure is performed first to obtain the desired occlusion in SF so that the patients tend to cooperate more in the post-surgical phase [4]. Additionally, it has been reported that the regional acceleratory phenomenon (RAP) effect and improvement of the perioral muscle balance of the SF approach will make the orthodontic treatment faster [5]. Moreover, the SF approach, which was introduced to address the disadvantages of a standard orthodontic procedure with pre-surgical, surgery, and post-surgery orthodontic treatment, has been shown to produce similarly successful outcomes with better patient satisfaction [6].

Recently, instead of routine orthodontics, clear aligner therapy such as Invisalign (Align Technology, Santa Clara, CA, USA) was introduced for orthodontic treatment. This emerging methodology matches the growing demand for more esthetic alternatives to the traditional braces and wires including patients with dentofacial deformities [7]. Through its software, ClinCheck (CC) (Align Technology, Santa Clara, CA, USA) facilitates the in-depth and accurate surgical planning required for the "surgery-first" approach, allowing the orthodontist and oral surgeon to envision the goals of the entire treatment plan before proceeding [8]. At first, this treatment was used for simple cases with minor orthodontic movements. Currently, with the advances in software and the development of materials, the clear aligner can be used in complex cases such as dentofacial deformities [9].

Typically, the advancement in digital technology enables us to achieve complete virtual planning. Advances in digital technology realize the paradigm shift from the 2D cephalometric radiographs to 3D virtual analysis and treatment planning using cone-beam computed tomography (CBCT) and computed tomography (CT) [10]. Using computeraided design and computer-aided manufacturing (CAD/CAM) technology to aid the intraoperative positioning of the maxillofacial complex by fabricating splints has been shown to be beneficial for oral and maxillofacial surgery [11]. Better still, these technological innovations have also impacted orthodontics, especially with the recent introduction of clear aligners such as Invisalign [12]. To meet the increased demand for more comfortable and esthetic treatment approaches, more and more patients have sought OGS together with clear aligner therapy such as Invisalign for corrective jaw surgery. Since the knowledge in this emerging interdisciplinary field is currently limited, the combined trend is also a new challenge to the orthodontist and the maxillofacial surgeon [8].

From the concept of a virtual laboratory, which can be directly applied at the university for not only educational purposes, but also for scientific research [13], recently, virtual methodology with the use of 3D modeling has become an emerging approach in the field of dental treatments, thanks to its efficiency and capability to reach the poorest areas of society for the prevention of oral diseases. For instance, a notable virtual approach was introduced in [14] with detailed methodology and analyses in the form of suitable context matrices to enable the virtual comparative analysis of biomaterials used in endodontic treatment. This kind of approach meets the concept of Dentistry 4.0, which is a combination of dentistry, dental engineering, and material engineering. Specifically, this concept has been realized in the design of prosthetic dental restorations with the significance of using cone-beam computed tomography (CBCT) in planning prosthetic treatment, and computeraided design and manufacturing methods (CAD/CAM) and additive manufacturing (AM) technologies for the manufacturing of prosthetic restorations [15] including selective laser sintering (SLS) for the typical case of dental implants [16] to enable the authors' new augmented holistic model of Industry 4.0 in advanced dental engineering. Materials and modern dental technologies have also been combined to realize material engineering methodology for research in clinical procedures and dental treatments. For example, in our prior work [17], we used acemannan, a polysaccharide extracted from aloe vera, as an implantable biomaterial for vital pulp therapy of immature permanent teeth induced continued root formation. The evaluation results using $\mathrm{CBCT}$ examination and $3 \mathrm{D}$ tooth reconstruction showed that by achieving an efficiency close to that of the traditional material (mineral trioxide aggregate (MTA)), acemannan is a promising low-cost biomaterial for partial pulpotomy treatment for immature permanent teeth requiring vital pulp therapy. Hence, with the use of modern dental technologies to enable advanced dental engineering 
for dental treatments, we can meet the concept of the Dentistry Sustainable Development (DSD) >2020 model consisting of three inseparable elements (i.e., Global Dental Prevention (GDP), Advanced Interventionist Dentistry 4.0 (AID 4.0), and the Dentistry Safety System (DSS)), with the hypothesis of the need to abandon interventionist dentistry in favor of the domination of dental prevention including a strategy for the active prevention of the spread of pathogenic microorganisms in the extreme cases of a global pandemic (e.g., SARS-CoV-2) [18].

With this in mind, to address the implication of the potential manual errors that existed in processing treatment of dentofacial deformity planning in the conventional approach with the use of the traditional 2D method [19], in this article, we present a fully digital scheme with virtual 3D modeling for the planning of surgical procedures as a novel modern dental engineering approach for dentofacial deformity treatment matching the concept of Dentistry 4.0 and DSD. To this end, in this paper, we analyze the merits of a combined protocol of surgical treatment with SFOA and orthodontic treatment with the Invisalign technique; present the novel concept of this hybrid method; then report our experience when conducting this integrated 3D technology-inspired approach for the dental deformity treatment with the obtained outcomes of typically complex cases. Specifically, this research article presents the integrated 3D technique by combining it with SFOA and clear aligner as a novel treatment method for the realization of an effective deformity treatment and then improvement of the patients quality of life. This is a competitive approach to the traditional conventional orthodontic - surgical treatment in which the novelty of this approach is connected with the adoption of 3D-based protocols in orthognathic surgery as well as digital orthodontic treatment as a method of virtual planning in the treatment of patients. The research then realizes a fundamental and significant change in clinical management as an enabler to the Sustainable Dentistry Development concept, which involves the use of 3D imaging and modeling as essential elements of Dentistry 4.0.

\section{Materials and Methods}

The present study follows the guidelines stated in the Declaration of Helsinki on Medical Protocol and Ethical Principles for Medical Research. Additionally, ethical approval was waived for this study by the institutional review board, due to its retrospective nature.

In this study, we retrospectively analyzed 13 patients diagnosed with various dentofacial deformities. All patients were treated by SF orthognathic surgery at Hanoi Medical University Hospital (Vietnam) and received Invisalign orthodontic treatment post-op. All patients agreed and signed consent showing understanding of the treatment objectives as well as acceptance of potential complications and consequences.

There were nine females and four males (13 patients in total) with ages ranging from 16 and 38 years old. All of these patients were referred to our center for malocclusion and esthetic concerns. No patient had temporo-mandibular joint disorders before surgery. Among them, six patients were diagnosed with skeletal class III, two patients were diagnosed with skeletal class II, three patients were diagnosed with a bimaxillary protrusion, and two patients were diagnosed with facial asymmetry.

Each patient underwent a thorough surgical and orthodontic examination together with a CT scan, extraoral and intraoral photographs (Figure 1), and intraoral scan using iTero Element (Align Technology, Santa Clara, CA, USA) (Figure 2). 


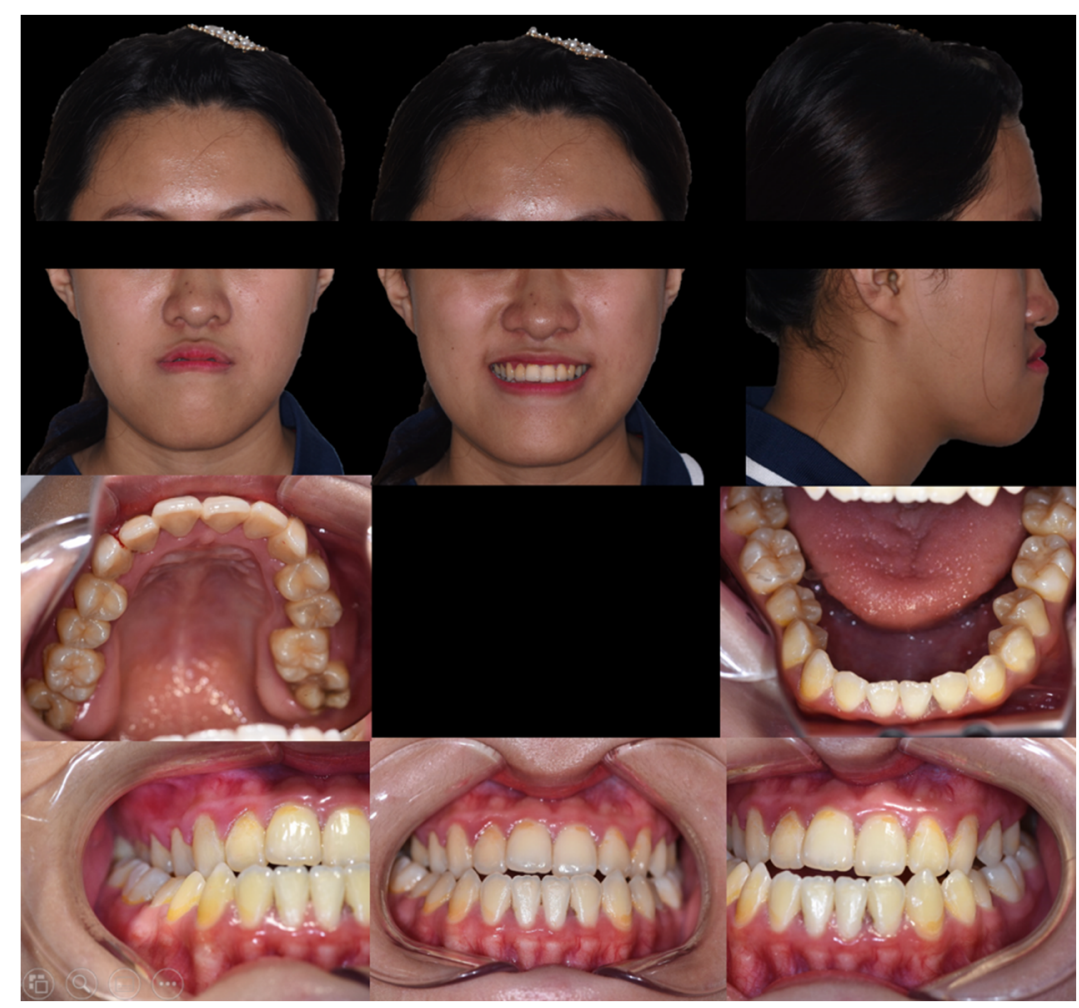

Figure 1. Intraoral and extraoral photos.

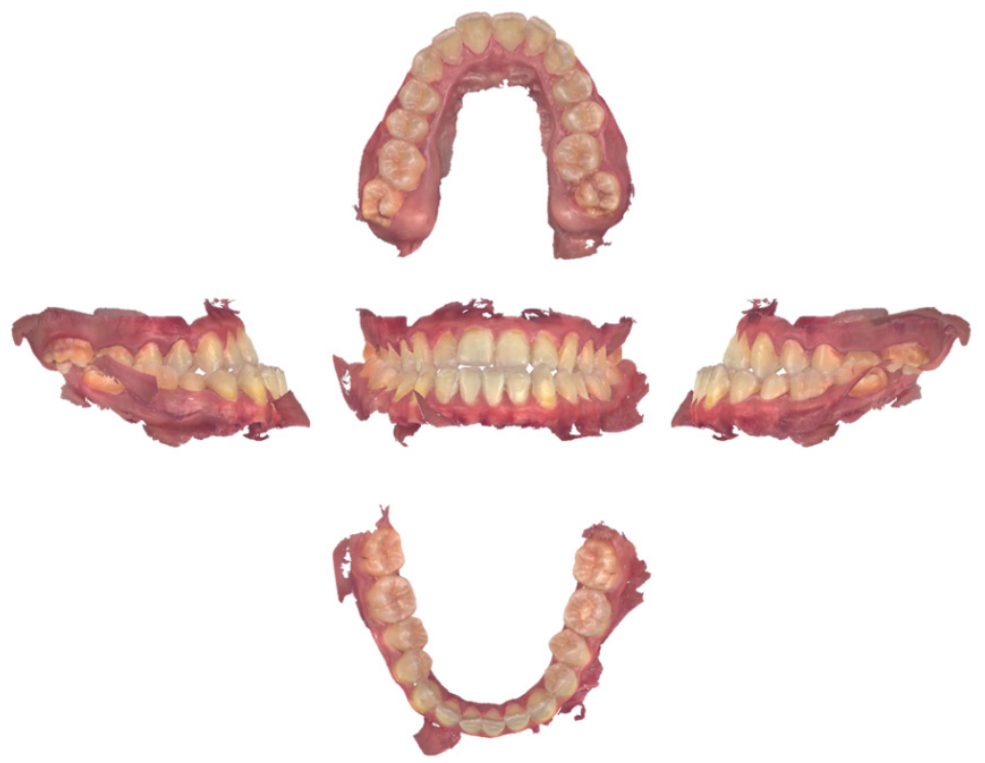

Figure 2. The intraoral scan using iTero Element.

\subsection{Digital Planning Workflow}

The ProPlan (Materialise, Belgium) software allowed for the superimposition between the photos, the 3D surgical movement, and the CT for visualization and simulation of the surgical results (Figure 3). The intraoral scans with ClinCheck software were used for the orthodontic treatment planning.

Specifically, the intraoral scanning of upper and lower dentitions for model creation of the orthodontic planning was performed using ClinCheck software. The files were also exported in STL for merging with the DICOM file from the CT data to analyze the 3D structure in surgical software. 3D visualization and planning enables a paradigm shift for 
the clinicians by conforming to the standard of traditional health care with a reduced level of manual errors.

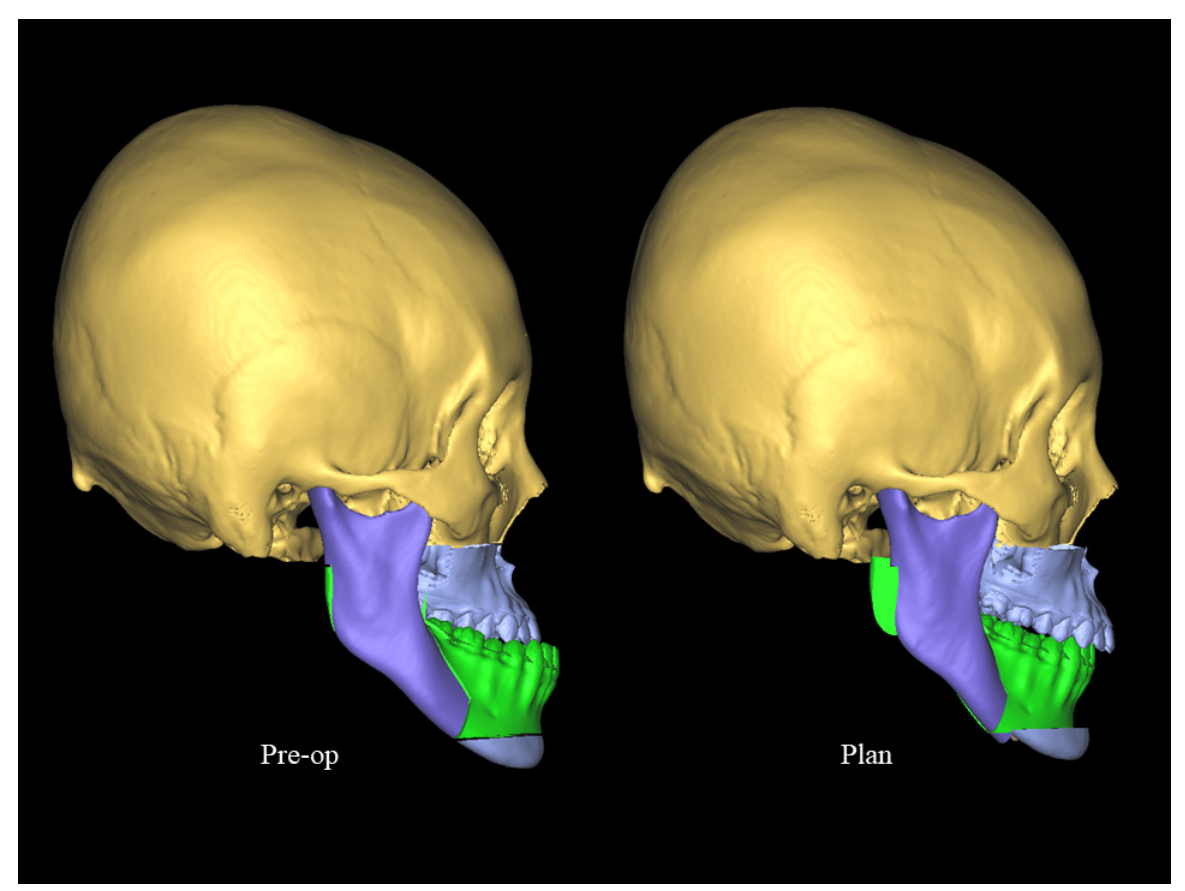

Figure 3. Three-dimensional (3D) virtual surgical plan.

The final occlusion was decided by the surgeon and orthodontist in a stone model relationship. The occlusion was then scanned by an Itero intraoral scanner in which occlusal contacts were analyzed by Itero digital occlusogram. The STL files were exported through which it is possible to design the surgical splint (Figure 4). Additionally, the final occlusion was integrated for use in the 3D surgical software to analyze the jaw movements, then correlate these repositions to the esthetic outcomes.

Analyzing the occlusal contact for the creation of a precise splint is fundamental since it guides the surgical fixation and occlusion. The post-operative occlusal relationship was carefully determined based on the post-surgical orthodontic treatment plan and slightly overcorrected the original part of the initial malocclusion. Post-surgery orthodontic treatment consists of interference elimination, compensatory deficiency, alignment, and adjustment of the dental arches, and the attainment of the correct occlusal relationship between the molars and canines. This procedure is carefully planned virtually through the ClinCheck software before the surgery (Figure 5).

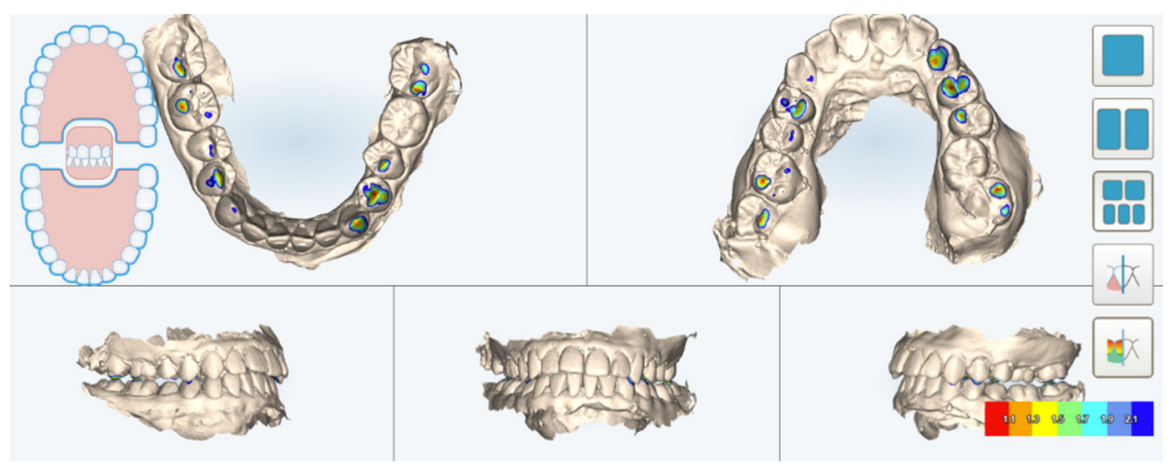

Figure 4. Post-operative occlusion was analyzed by digital occlusogram. 

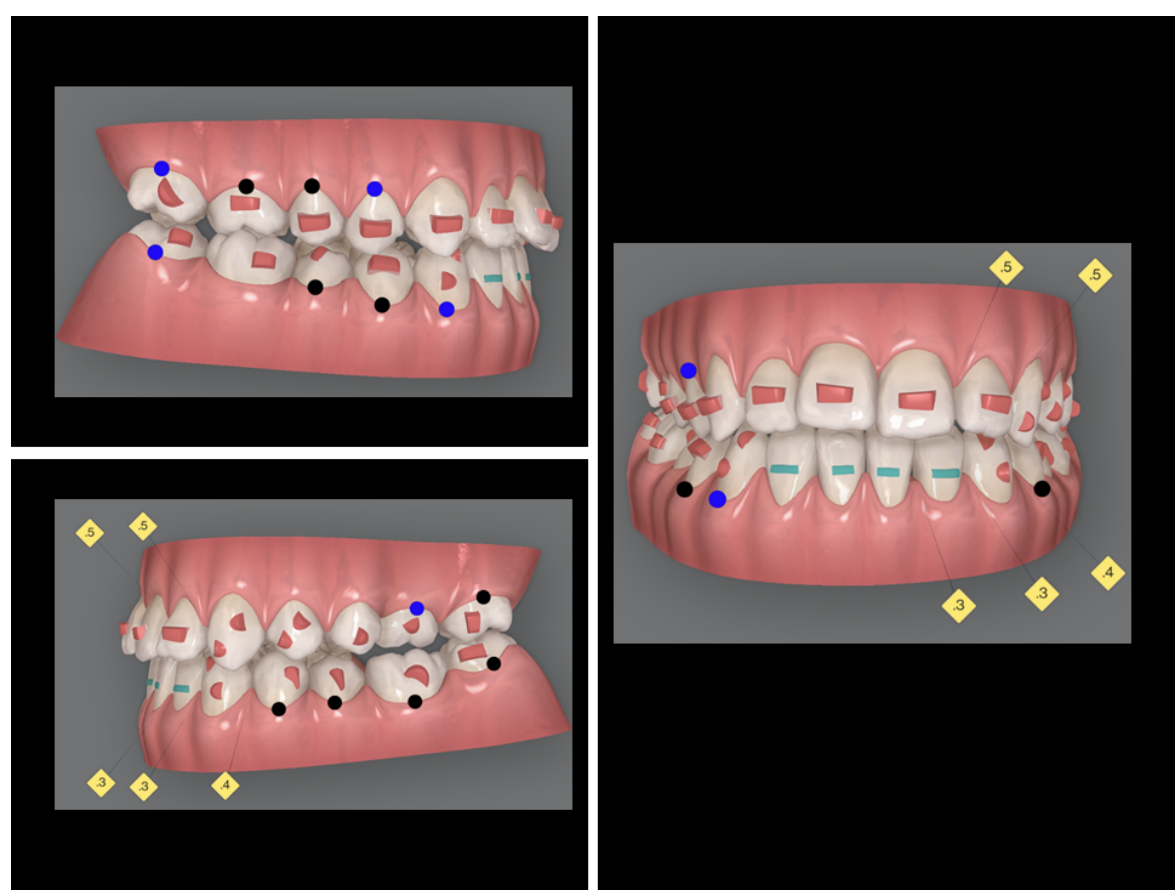

Figure 5. Three-dimensional virtual orthodontic plan.

\subsection{Treatment Progress}

The aligners could be fabricated according to the ClinCheck plan before surgery depending on the orthodontist's decision. Attachments were bonded using a template so that patients could use aligners right after surgery.

\subsection{Surgery}

All patients were operated by the first author. Three patients diagnosed with bimaxillary protrusion were treated by subapical osteotomy of the upper and lower jaw with four first bicuspid extractions. All other 10 patients were submitted to bilateral sagittal split osteotomy and Le Fort I. Genioplasty was conducted as needed in some patients to enhance the patient's esthetic. Since the patient would not be wearing fixed appliances during orthodontic treatment, intermaxillary fixation was supplied by eight temporary anchorage devices (TADs) inserted between the molars and premolars and between the canines and lateral incisors in all quadrants. Intermaxillary elastics were worn from the TADs for about one month after the surgery to counter any surgical relapse.

\subsection{Post-Surgery}

After the surgery as planned was undertaken, a CT scan was taken to show that the condylar positions were stable and unchanged at pre- and post-surgery (Figure 6). Postsurgical orthodontic treatment, planned through ClinCheck, includes wearing aligners one week before surgery and approximately one week after surgery. To support and guide the surgically corrected occlusion, it is usually necessary to employ postsurgical elastics. The elastics are often made via orthodontic TADs (Figure 7), directly into the notches on the aligner tray or bonded buttons. Note that it may not be efficient to use the "post-jump" aligners by Invisalign manufacturers as predicting the precise postsurgical occlusion is challenging. In this case, the surgeon or orthodontist can scan the dentition by two weeks postoperatively to update the occlusion and fabricate refinement aligners.

The aligners are changed every three to five days to expect the RAP effect post-surgery. After that, the aligners were changed every one to two weeks. The total orthodontic treatment lasted between six and 18 months. 


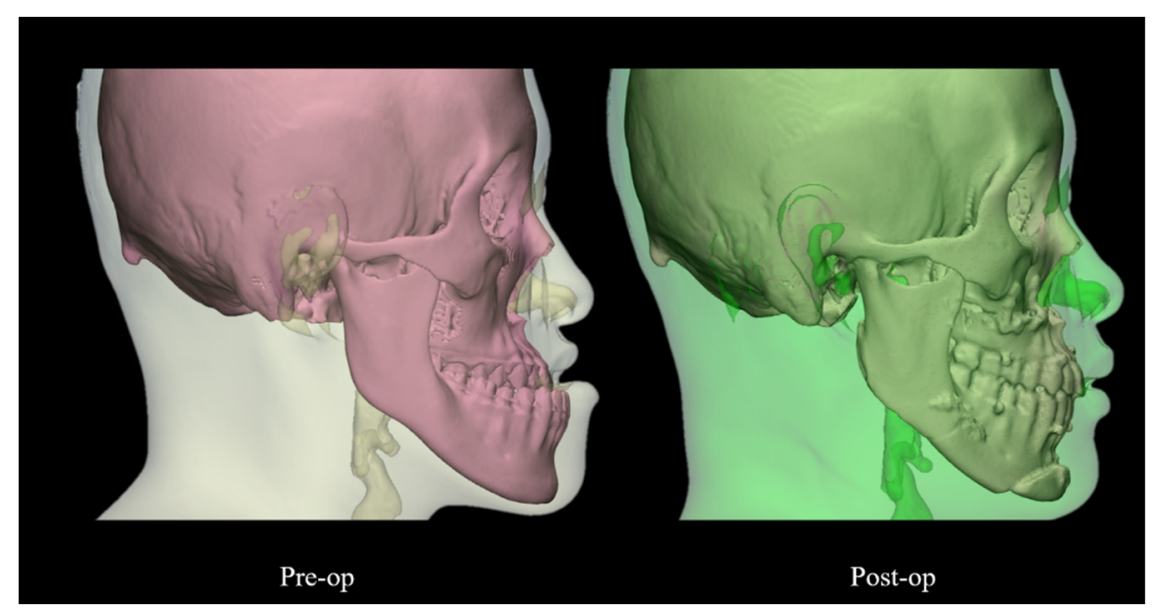

Figure 6. Post-operative CT was taken to confirm the condylar position, bone movement according to the treatment plan, and hardware positions.

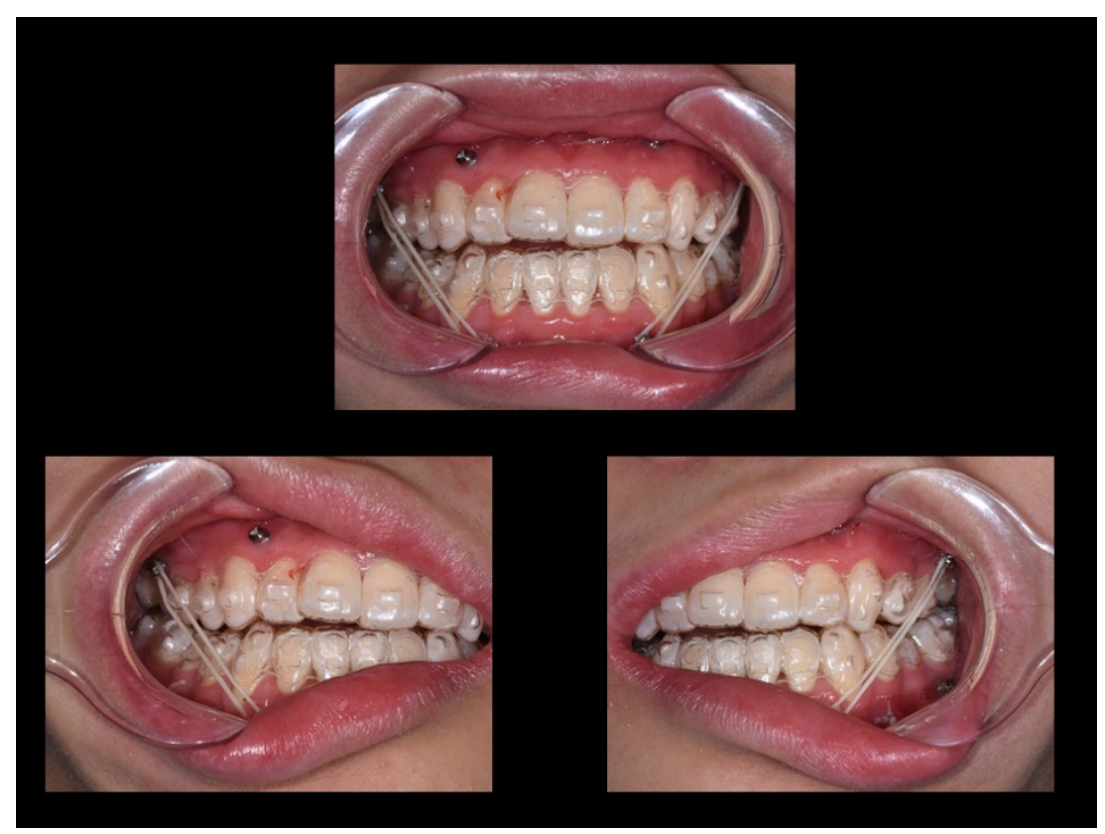

Figure 7. Elastic training through TADs.

\section{Results}

From the obtained post-treatment records, we demonstrate that by using the described combined methodology, the treatment goals were attained with good occlusal and esthetic results (Figure 8). Additionally, Table 1 shows the detailed 3D cephalometric measurements before and after performing the combined orthognathic and orthodontic treatment in the exemplified case presented in the paper.

Typically, facial symmetry was achieved with the dental midline coincided with the facial midline, resulting in ideal overbite and overjet with a Class I buccal occlusion. No patient was referred with serious postoperative complications or TMJ discomfort. All patients were satisfied with the final esthetic outcomes and resolution of their complaints. 


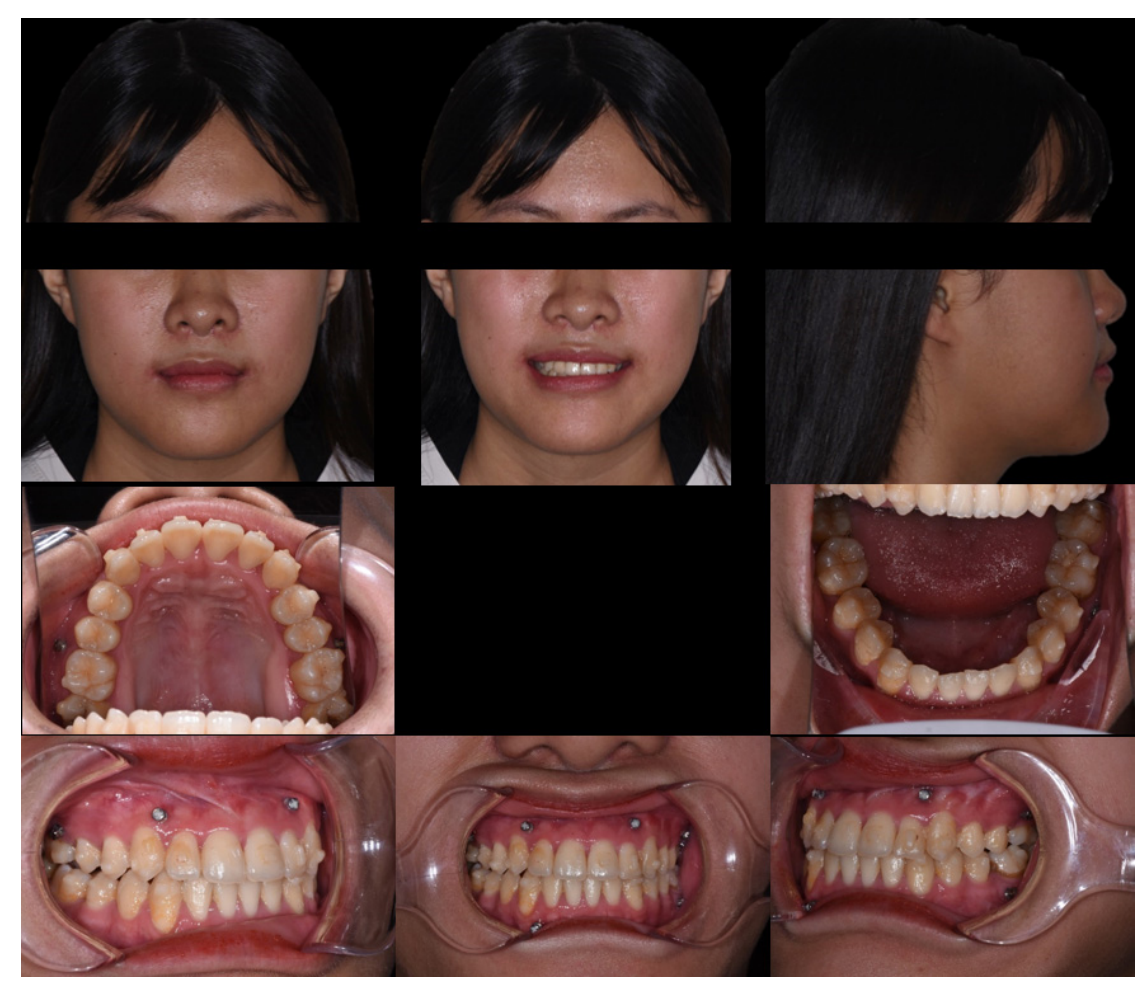

Figure 8. Intraoral and extraoral photos at the end of the treatment.

Table 1. 3D cephalometric measurements before and after performing the combined orthognathic and orthodontic treatment.

\begin{tabular}{|c|c|c|}
\hline Parameters & Pre & Post \\
\hline \multicolumn{3}{|l|}{ Soft Tissue } \\
\hline Soft tissue convexity angle (N-Sn-Pg) & -2.93 & 9.86 \\
\hline Nasolabial angle & 132.74 & 122.71 \\
\hline Upper lip length (Sn-Sto) & 17.34 & 21.42 \\
\hline Lower lip length (SI-Sto) & 47.19 & 41.62 \\
\hline Upper lip to E line & -7.39 & -3.48 \\
\hline Lower lip to E line & 4.26 & -0.99 \\
\hline \multicolumn{3}{|l|}{ Skeletal } \\
\hline Upper third of facial height (N-ANS) & 54.37 & 55.25 \\
\hline Lower third of facial height (ANS-Me) & 70.09 & 68.65 \\
\hline N-ANS/ANS-Me (UAFH/LAFH) & 0.78 & 0.8 \\
\hline Facial convexity (N-A/A-Pog) & -15.34 & 1.35 \\
\hline SNA & 81.56 & 85.39 \\
\hline SNB & 90.48 & 84.54 \\
\hline ANB & -9.08 & 1.24 \\
\hline A-N perpendicular & -3.93 & 0.1 \\
\hline B-N perpendicular & 11.26 & 3.31 \\
\hline Pog-N perpendicular & 9.25 & 0.54 \\
\hline Menton deviation (Me-MSP) & 7.14 & 1.22 \\
\hline Mandibular plane angle (FH/MP) & 31.26 & 31.63 \\
\hline
\end{tabular}


Table 1. Cont.

\begin{tabular}{ccc}
\hline Parameters & Pre & Post \\
\hline Dental & & \\
\hline Upper incisors inclination & & 112.41 \\
\hline U1/SN & 123.78 & 7.74 \\
\hline U1-NA & 12.56 & 27.02 \\
\hline U1/NA & 42.23 & \\
\hline Lower incisor inclination & & 35.3 \\
\hline L1/MP & 70.72 & 23.51 \\
\hline L1-NB & 4.16 & 129.81 \\
\hline L1/NB & 17.63 & \\
\hline Interincisal angle & & 11.27 \\
\hline U1/L1 & 129.07 \\
\hline Occlusal plane angle & & \\
\hline FH-Occlusal plane & 8.65 & \\
\hline
\end{tabular}

\section{Discussion}

The obtained outcomes show that the combination of Invisalign and SF, without adversely affecting the patient's quality of life, offers a minimally invasive alternative to the clinician for managing severe dentoskeletal discord. Kankam et al. stated that when implementing clear aligner therapy instead of traditional fixation devices, there is no significant effect on postoperative edema and perioperative outcomes [20].

The combination of orthognathic surgery and clear-aligner therapy (e.g., Invisalign) is a novel idea, but with limited evidence thus far $[9,20]$. To date, knowledge of clear aligners with OGS is minimal, with most being case reports [8,21-24]. For instance, when searching for "orthognathic and Invisalign" or "orthognathic and clear aligner" in Scopus and PubMed, only five publications were returned.

The selection criteria for the SF highly depend on the preference and experience of the orthodontist and the surgeon. Furthermore, for the SF, no consensus on the indications and contraindications is declared [25]. From early investigations, SF is indicated in mild cases when no tooth extraction is included, there is little or no lateral discrepancy, and there are at least three occlusal contact points between the arches [26]. For open bite and facial asymmetry cases, formerly, these cases would be discouraged by the SF [27]. This is also true for SF combined with clear aligner therapy as almost all published papers present skeletal class III malocclusion [9,21-23]. At present, the indication barriers for the SF and clear aligner therapy are constantly changing and may be overcome by technological advancements. In this study, the patients were successfully treated by a novel combined approach for various dentofacial deformities such as skeletal class III, class II, extraction involved, facial asymmetry, and so on.

When this combined approach is implemented, several challenges must be considered. Since the transparent aligner is removable and usually needs to be worn for 20-22 h daily, successful treatment with Invisalign depends on good patient compliance [12]. An approach to intermaxillary fixation (IMF) is a typical intraoperative challenge. Fixed orthodontic appliances consisting of bands, brackets, and arch wires facilitate wire IMF. Nevertheless, a different approach is needed for Invisalign patients. One such technique is to apply the Erich arch bars before the fixation of the opposing arches with wires. However, the described approach with TADs remains a favorable tool since it is associated with improved oral hygiene related to arch bars and reduced procedural time [9]. Despite this, the use of TAD has its disadvantages, with root damage and loose screws being the most popular complications. With the aid of the CAD/CAM technology, another novel technique for relocation and fixation 
using the Clear Aligner Orthognathic Splint (CAOS) has been created [28]. The CAOS offers complete coronal coverage of the dental arches and high adaptability, allowing fixation and repositioning of the maxillomandibular complex without the need for wires. However, this technique requires more laboratory work and may not be cost-effective. Therefore, we prefer the TAD techniques, as they are also used in wearing elastic post-operation and may be needed by orthodontics to control some tooth movement.

In SF, conducting the orthodontic treatment is faster for the postsurgical RAP [5]. The metabolic changes during the healing period result in an increased bone turnover with a shorter post-surgery treatment time and a faster orthodontic movement. To take advantage of RAP in the SF approach for post-operative rapid tooth movement, clear aligners should be placed in the patient's mouth as early as possible. In this study, the patients used aligners early at seven days post-surgery and changed aligners at a shorter duration (every three to five days). No post-operative splint and intermaxillary fixation were used after surgery to facilitate immediate tooth movement and improve the patients' hygiene and comfort. In the event of a transition to an undesired bite, light elastics or occlusal adjustments were used to stabilize the jaw position.

For such complex treatments, the accuracy of both surgical and orthodontic planning takes a key role in the success of the treatment. The digital advance paves the way for the virtual visualization of the actual patient that represents all the patient's tissues including teeth, soft tissue, and bone in a single 3D model. Hence, we can perform simulation and virtual planning for both orthodontic treatments and surgery. Moreover, the innovation in the advent of CAD/CAM technology opens the possibility of ensuring remarkable and reproducible results in terms of the functional and anatomical perspectives [29]. Digital technology also offers a solution for follow-up of the orthodontic treatment by using Itero Timelapse and Progress assessment tools that can track the tooth movement (Figure 9). This technology is useful for the orthodontist to detect any off-track movement and plan refinement or additional aligners if needed, especially in challenging treatments like dentofacial deformity cases.

Though the present study had a retrospective design with a limited number of subjects involved, it showed that clear aligners (e.g., Invisalign) can be efficiently used for patients undergoing SF orthognathic surgery. This approach then realizes a novel concept with early promising and positive pilot outcomes, but to confirm these findings, randomizedcontrolled trials are needed for further studies.

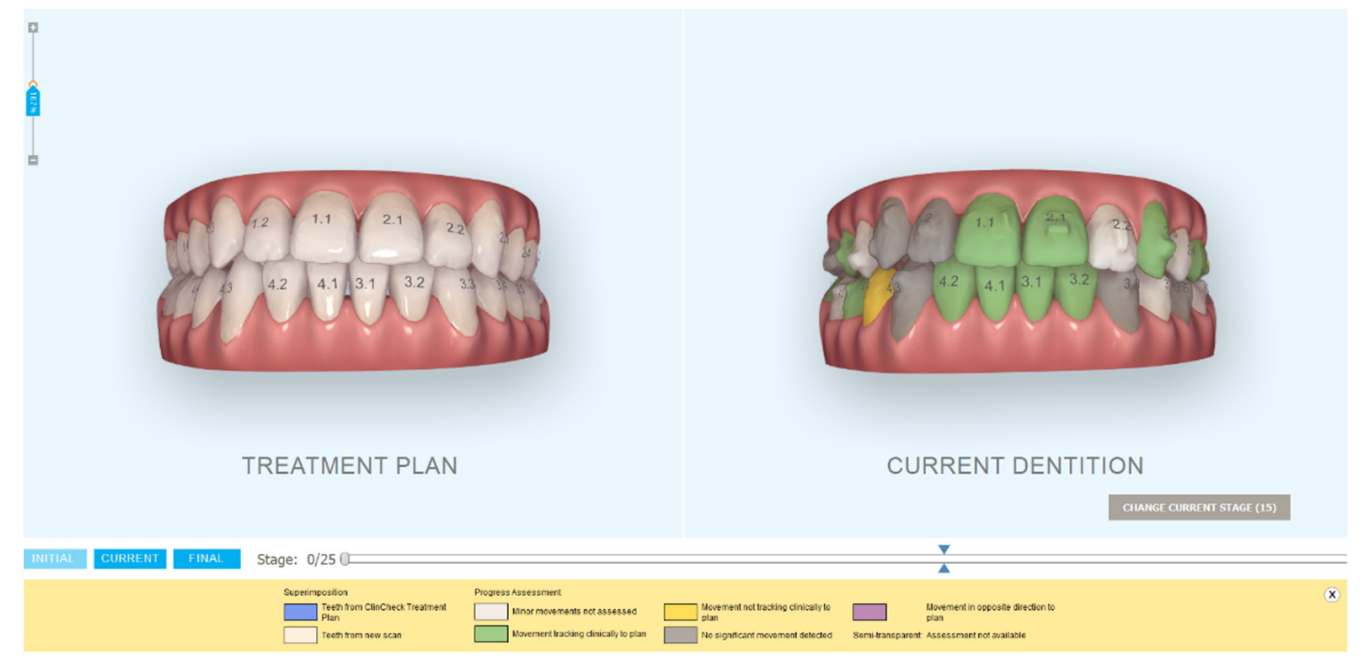

Figure 9. Invisalign progress assessment allows the orthodontist to digitally follow up the treatment case.

\section{Conclusions}

With the recent digital revolution, the treatment of complex cases can benefit from 3D technology (e.g., the use of $\mathrm{CT}$, intraoral scanners, and CAD/CAM systems). Given that 
it is necessary to implement a virtual methodology to design surgical procedures for the practical realization of a novel modern dental engineering approach for dentofacial deformity treatment, in this article, we present an advanced digital 3D technology in combined SF orthognathic and clear aligner orthodontic therapy as a fully digitalized treatment methodology. Moreover, as described in the present study, this novel combined approach includes the use of digital software for efficient planning and assists in both surgical and orthodontic treatment, which is promising and more accurate compared to the conventional approach.

The final esthetic outcomes from that recorded of all the involved patients showed that with the present digital 3D technology, surgery first and clear aligner therapy (e.g., Invisalign) can be performed together to combine the merits of both for the realization of a more comfortable treatment with a quicker entire treatment time and a better social esthetic impact, then improve the quality of life for dentofacial deformity patients.

Overall, the resulting implications for the planning of surgical procedures described in this article show that the virtual techniques based on CBCT/CT imaging and virtual planning of treatments in the field of craniofacial surgery ensure the precision of subsequent treatments in reality and avoid errors in removing dentofacial deformities as much as possible. The present approach also covers important issues in the treatment of patients with dentofacial deformities to obtain an ideal facial esthetic with good functioning. Though the number of treatments cannot be large due to the nature and complexity of the combined treatment (considering the type and frequency of this type of disease, and on the other hand, the extent of the dysfunction from the patients' psychological and medical point of view should be considered), the evaluation results in general and the exemplified treatment, in particular, demonstrate that the present combined technique of SF and clear aligner together with the Advanced Digital 3D Technology acts as an innovative and efficient approach for dentofacial deformity treatment. We then shed new light on the realization of practical implementation of the novel integrated treatment method using 3D technology with the use of 3D imaging and modeling as a promising development trend of dentistry that fits into the context of Dentistry 4.0 as a key enabler to the concept of sustainable dentistry development.

Author Contributions: Conceptualization, M.T.N. and T.T.V.; Methodology, M.T.N., T.T.V. and Q.N.N.; Software M.T.N. and Q.N.N.; Validation, M.T.N., Q.N.N. and T.T.V.; Formal analysis, M.T.N. and Q.N.N.; Investigation, M.T.N. and Q.N.N.; resources, M.T.N. and Q.N.N.; writing—original draft preparation, M.T.N. and T.T.V.; writing—review and editing, Q.N.N. and M.T.N.; visualization, M.T.N. and Q.N.N.; Supervision, Q.N.N. and M.T.N.; Project administration, Q.N.N. All authors have read and agreed to the published version of the manuscript.

Funding: The authors would like to thank Hanoi Medical University and Chulalongkorn University for supporting and funding us in conducting this research.

Institutional Review Board Statement: Ethical approval was waived for this study by the institutional review board, due to its retrospective nature.

Informed Consent Statement: Informed consent was obtained from all subjects involved in the study.

Data Availability Statement: Not applicable.

Conflicts of Interest: The authors declare no conflict of interest.

\section{References}

1. Grubb, J.; Evans, C. Orthodontic management of dentofacial skeletal deformities. Clin. Plast. Surg. 2007, 34, 403-415. [CrossRef]

2. Luther, F.; Morris, D.O.; Hart, C. Orthodontic preparation for orthognathic surgery: How long does it take and why? A retrospective study. Br. J. Oral. Maxillofac. Surg. 2003, 41, 401-406. [CrossRef]

3. Nagasaka, H.; Sugawara, J.; Kawamura, H.; Nanda, R. "Surgery first" skeletal Class III correction using the Skeletal Anchorage System. J. Clin. Orthod. 2009, 43, 97-105.

4. Saghafi, H.; Benington, P.; Ayoub, A. Impact of orthognathic surgery on quality of life: A comparison between orthodontics-first and surgery-first approaches. Br. J. Oral. Maxillofac. Surg. 2020, 58, 341-347. [CrossRef]

5. Liou, E.J.; Chen, P.H.; Wang, Y.C.; Yu, C.C.; Huang, C.S.; Chen, Y.R. Surgery-first accelerated orthognathic surgery: Postoperative rapid orthodontic tooth movement. J. Oral. Maxillofac. Surg. 2011, 69, 781-785. [CrossRef] 
6. Yang, L.; Xiao, Y.D.; Liang, Y.J.; Wang, X.; Li, J.Y.; Liao, G.Q. Does the Surgery-First Approach Produce Better Outcomes in Orthognathic Surgery? A Systematic Review and Meta-Analysis. J. Oral. Maxillofac. Surg. 2017, 75, 2422-2429. [CrossRef]

7. Azaripour, A.; Weusmann, J.; Mahmoodi, B.; Peppas, D.; Gerhold-Ay, A.; Van Noorden, C.J.; Willershausen, B. Braces versus Invisalign(R): Gingival parameters and patients' satisfaction during treatment: A cross-sectional study. BMC Oral. Health 2015, 15, 69. [CrossRef] [PubMed]

8. Chang, J.; Steinbacher, D.; Nanda, R.; Uribe, F. "Surgery-First" Approach with Invisalign Therapy to Correct a Class II Malocclusion and Severe Mandibular Retrognathism. J. Clin. Orthod. 2019, 53, 397-404. [PubMed]

9. Amodeo, G.; Meuli, S.; Carboni, A.; Brugnami, F.; Marrocco, S.; Orsini, R.; Scopelliti, D. Surgery First and Invisalign System: Combined Digital Approach. J. Craniofac. Surg. 2020, 31, 1681-1686. [CrossRef] [PubMed]

10. Aboul-Hosn Centenero, S.; Hernandez-Alfaro, F. 3D planning in orthognathic surgery: CAD/CAM surgical splints and prediction of the soft and hard tissues results-our experience in 16 cases. J. Craniomaxillofac. Surg. 2012, 40, 162-168. [CrossRef] [PubMed]

11. Lonic, D.; Pai, B.C.; Yamaguchi, K.; Chortrakarnkij, P.; Lin, H.H.; Lo, L.J. Computer-Assisted Orthognathic Surgery for Patients with Cleft Lip/Palate: From Traditional Planning to Three-Dimensional Surgical Simulation. PLoS ONE 2016, 11, e0152014. [CrossRef]

12. Wong, B.H. Invisalign A to Z. Am. J. Orthod. Dentofacial. Orthop. 2002, 121, 540-541. [CrossRef]

13. Honysz, R.; Dobrzanski, L. Virtual laboratory methodology in scientific researches and education. J. Achiev. Mater. Manuf. Eng. 2017, 2, 76-84. [CrossRef]

14. Dobrzańska, J.; Dobrzański, L.B.; Gołombek, K.; Dobrzanski, L.; Dobrzańska-Danikiewicz, A. Virtual Approach to the Comparative Analysis of Biomaterials Used in Endodontic Treatment. Processes 2021, 9, 926. [CrossRef]

15. Dobrzanski, L.; Dobrzański, L.B. Dentistry 4.0 Concept in the Design and Manufacturing of Prosthetic Dental Restorations. Processes 2020, 8, 525. [CrossRef]

16. Dobrzanski, L.; Dobrzański, L.B.; Achtelik-Franczak, A.; Dobrzańska, J. Application Solid Laser-Sintered or Machined Ti6Al4V Alloy in Manufacturing of Dental Implants and Dental Prosthetic Restorations According to Dentistry 4.0 Concept. Processes 2020, 8, 664. [CrossRef]

17. Vu, T.; Nguyen, M.; Sangvanich, P.; Nguyen, Q.; Thunyakitpisal, P. Acemannan Used as an Implantable Biomaterial for Vital Pulp Therapy of Immature Permanent Teeth Induced Continued Root Formation. Pharmaceutics 2020, 12, 644. [CrossRef]

18. Dobrzanski, L.; Dobrzański, L.B.; Dobrzańska-Danikiewicz, A.; Dobrzańska, J. The Concept of Sustainable Development of Modern Dentistry. Processes 2020, 8, 1605. [CrossRef]

19. Nguyen, M.; Visuttiwattanakorn, S.; Wongsirichat, N. Prediction of pharyngeal airway changes in skeletal class III deformity patients after orthognathic surgery. J. Oral Maxillofac. Surg. Med. Pathol. 2015, 27. [CrossRef]

20. Kankam, H.; Madari, S.; Sawh-Martinez, R.; Bruckman, K.C.; Steinbacher, D.M. Comparing Outcomes in Orthognathic Surgery Using Clear Aligners Versus Conventional Fixed Appliances. J. Craniofac. Surg. 2019, 30, 1488-1491. [CrossRef]

21. Boyd, R.L. Surgical-orthodontic treatment of two skeletal Class III patients with Invisalign and fixed appliances. J. Clin. Orthod. 2005, 39, 245-258.

22. Marcuzzi, E.; Galassini, G.; Procopio, O.; Castaldo, A.; Contardo, L. Surgical-Invisalign treatment of a patient with Class III malocclusion and multiple missing teeth. J. Clin. Orthod. 2010, 44, 377-384. [PubMed]

23. Pagani, R.; Signorino, F.; Poli, P.P.; Manzini, P.; Panisi, I. The Use of Invisalign(R) System in the Management of the Orthodontic Treatment before and after Class III Surgical Approach. Case Rep. Dent. 2016, 2016, 9231219. [CrossRef] [PubMed]

24. Womack, W.R.; Day, R.H. Surgical-orthodontic treatment using the Invisalign system. J. Clin. Orthod. 2008, 42, 237-245.

25. Huang, C.S.; Hsu, S.S.; Chen, Y.R. Systematic review of the surgery-first approach in orthognathic surgery. Biomed. J. 2014, 37, 184-190. [CrossRef] [PubMed]

26. Baek, S.H.; Ahn, H.W.; Kwon, Y.H.; Choi, J.Y. Surgery-first approach in skeletal class III malocclusion treated with 2-jaw surgery: Evaluation of surgical movement and postoperative orthodontic treatment. J. Craniofac. Surg. 2010, 21, 332-338. [CrossRef] [PubMed]

27. Hernandez-Alfaro, F.; Guijarro-Martinez, R.; Peiro-Guijarro, M.A. Surgery first in orthognathic surgery: What have we learned? A comprehensive workflow based on 45 consecutive cases. J. Oral. Maxillofac. Surg. 2014, 72, 376-390. [CrossRef]

28. Caminiti, M.; Lou, T. Clear Aligner Orthognathic Splints. J. Oral. Maxillofac. Surg. 2019, 77, 1071.e1-1071.e8. [CrossRef]

29. Vale, F.; Scherzberg, J.; Cavaleiro, J.; Sanz, D.; Caramelo, F.; Malo, L.; Marcelino, J.P. 3D virtual planning in orthognathic surgery and CAD/CAM surgical splints generation in one patient with craniofacial microsomia: A case report. Dental Press J. Orthod. 2016, 21, 89-100. [CrossRef] 